\title{
Intelligent Automatic Deburring System by Integrating Palletizing Robot with Image and Vibration Sensors
}

\author{
Yung-Fu Hsieh, Jhan-Hong Ye, Nan-Jyue Wu, and Quang-Cherng Hsu* \\ Department of Mechanical Engineering, National Kaohsiung University of Science and Technology, \\ No. 415, Jiangong Rd., Sanmin Dist., Kaohsiung City 80778, Taiwan \\ (Received December 14, 2020; accepted February 12, 2021)
}

Keywords: palletizing robot, machine vision, deburring, vibration sensor

The edge of the contour of a stamping part is burred because of the shearing phenomenon when punching or blanking. To detect burrs, manual visual inspection is often used, which is time-consuming and inefficient, making a systematic detection method necessary. In this study, we developed a palletizing robot with a three-axis vibration sensor, vision sensor, gripper, electric deburring tool, and machine vision software that can detect the burring of punching holes for stamping parts. The vibration sensor is used to monitor the vibrations during workpiece clamping, grinding, and placing. If the vibration signal exceeds the threshold value, the system immediately stops. Integrating image and vibration sensors into the proposed palletizing robot enabled effective and intelligent detection, deburring, and monitoring processes.

\section{Introduction}

Burr defects often occur during manufacturing processes such as machining, stamping, and casting, which need to be removed before precision assembly. Rejc et al. ${ }^{(1)}$ measured gray-iron grates by combining laser triangular displacement sensors and industrial Selective Compliance Assembly Robot Arms and adopting a noncontact size measurement method. They developed a novel polynomial contour approximation method for repairing scan data and calculating the coordinates of metal burrs. Their results indicated that this method can measure 88 points on a gray-iron grate with a precision of $0.3 \mathrm{~mm}$ within $45 \mathrm{~s}$, and thus this method is sufficient for robots to deburr gray-iron grates.

In mass production, operators program deburring robots by using "teach" or "offline" programming to deburr metals. To solve the problems encountered by these two programming methods, Princely and Selvaraj ${ }^{(2)}$ introduced a vision-guided robotic system to deburr workpieces. The system obtains the two-dimensional shapes of all workpieces and automatically generates a robotic arm program according to the workpiece shape data and processing conditions that are collected. Their experimental results indicated that this method is effective in deburring operations.

Kuss et al. ${ }^{(3)}$ proposed a deburring process that uses adaptive and automated robotic arms. This process uses manufacturing tolerance to obtain the changes in the geometry of workpieces.

*Corresponding author: e-mail: hsuqc@nkust.edu.tw https://doi.org/10.18494/SAM.2021.3220 
Subsequently, the geometric shapes of the workpieces are generally matched using the iterative closest point method to compare the point clouds created from the manufactured workpieces and those generated under various levels of tolerance. Then, the most similar models are selected for subsequent deburring path planning and positioning. Their experimental results revealed that this method can be effectively applied in industry to enhance deburring quality.

After chip processing, the raised edge or small piece of material attached to the workpiece is called a burr. ${ }^{(4)}$ Shahid et al. proposed a hybrid method for vision-based surface coverage measurement in 2019. ${ }^{(5)}$ This method is based on morphological operations and Boykov graph cut segmentation. Their results indicated that images have better image segmentation results with nonuniform illumination. Image acquisition devices can be used to capture the contours of objects and divide the edges of the deburring portions through image segmentation, and thus the processing path for deburring is automatically generated. ${ }^{(6)}$

Many researchers have proposed methods to improve the deburring quality or make the entire deburring process more intelligent and flexible. ${ }^{(7-11)}$ However, a systematic approach combining the design of a four-axis robot, machine vision architecture, deburring method, and vibration monitoring is still needed. Taking the stamping process as an example, the edge of the contour of the billet is burred because of shearing when punching or blanking. To detect burrs, manual visual inspection is often used but this method is time-consuming and inefficient, making it necessary to develop a systematic detection method. In this study, we developed a palletizing robot with a three-axis vibration sensor, image sensor, gripper, electric deburring tool, and machine vision software that can detect the burring of punching holes and deburr automatically for stamping parts under vibration safety monitoring.

\section{Research Methods}

\subsection{Performance assessment and test method}

This study uses ISO 9283 (i.e., Manipulating industrial robots-Performance criteria and related test methods) ${ }^{(12)}$ as the pose repeatability standard for assessing robot performance. The developed palletizing robot is taught to move its robotic arm to several path points. Repeated movements are made to assess the performance of the robotic arm by using performance indicators.

\subsection{Mechanism design principles of palletizing robot}

In essence, the palletizing robotic arm is designed using two parallelograms. The terminal axis is always perpendicular to the ground, that is, $\theta_{1}+\theta_{2}+\theta_{3}=180^{\circ}$. The basic structure of the robotic arm is shown in Fig. 1. The palletizing robotic arm is an upright and four-axis jointlike robotic arm. Each joint is driven by a motor combined with a reducer to move and turn the robotic arm. For the connecting rod design, each connecting rod contains points BCD. Points $\mathrm{OABC}$ make up a parallelogram. The mechanism magnification coefficient is $\mathrm{CD} / \mathrm{BC}>1$, where an overly small magnification coefficient exposes the robotic arm to an excessive amount of interference, whereas an overly large magnification coefficient creates an excessive amount 


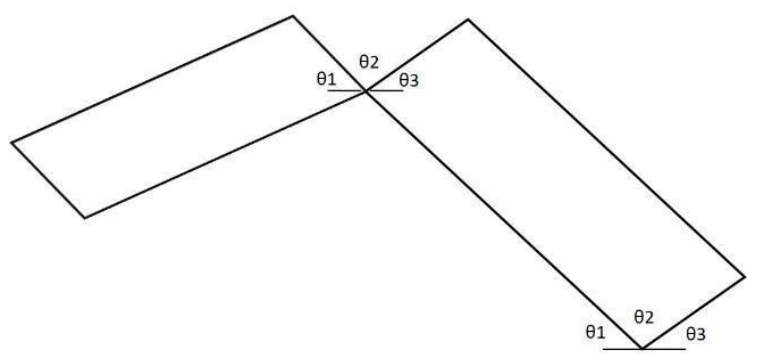

Fig. 1. Basic structure of the palletizing robotic arm.

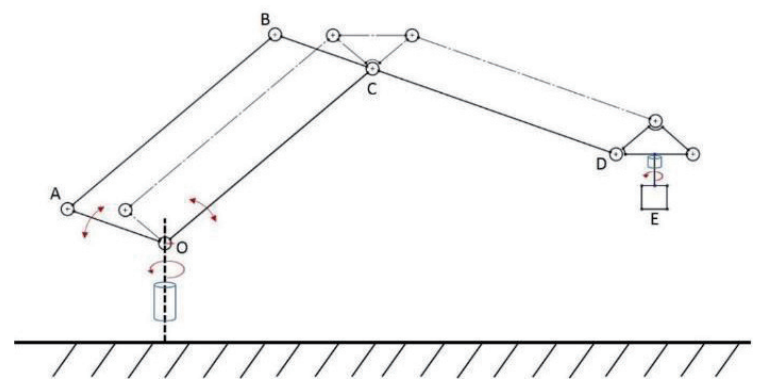

Fig. 2. (Color online) Movement direction of each joint.

of inertia when the robotic arm is turned. Connecting rods OA and OC primarily control the movements of terminal point $\mathrm{E}$. The direction of the movement of each joint is shown in Fig. 2. This type of robotic arm features a simple structure and a high loading capacity.

The robotic arm introduced in this study has an effective load of 3-5 kgf and shortest and longest travel distances of 250 and $700 \mathrm{~mm}$, respectively, where the travel distance of the arm is defined as the distance between the center of the original axis and the center of the final axis. To prevent the components from interfering in the distance-measuring process, a threedimensional assembly diagram is used. ${ }^{(13)}$ Each component is named (Fig. 3) to enable users to conveniently modify and assemble components.

According to the standard performance defined in the design, the shortest and longest working distances are set as 250 and $700 \mathrm{~mm}$, respectively. To prevent the robotic arm from overextending itself and thus become unable to retract to its original location, the smallest and largest angles between the lower arm and the base are set as $30^{\circ}$ and $80^{\circ}$, respectively. The lengths of the remaining connecting rods are set as variables. The shortest and longest working distances are measured using a graphic design software program, and the measurements are made under the condition that the components do not collide or interfere with one another when the angle between the lower arm and base is $30^{\circ}$ or $80^{\circ}$.

The robotic arm design was changed four times after factors such as the center of gravity of the overall structure, the assembly of the components, material costs, and component interferences were considered. Diagrams of the overall robotic arm design at each stage (i.e., Types 1-5) are presented in Fig. 4.

In Type 1, the center of gravity of the robotic arm was shifted to one side because most of the components were lined up on that side. To ensure that the center of gravity of the robotic arm was at the center of the robotic arm platform, the design was changed to Type 2. To match the components provided by the manufacturer, such as the reducer, motor, and gripper, Type 2 was changed into Type 3. Because the material cost for Type 3 was excessively high, to lower the cost, the rod components exposed to less force were replaced to produce Type 4 . Because the upper and lower arms in Type 4 had an embedded design that led to components interfering with one another when the shortest and longest working distances were set, the upper arm was made arc-like and the lower arm groove was extended. Type 5 was the final robotic arm design, which eliminates the interference problem encountered in Type 4 (Figs. 5 and 6). Type 6 was the final configuration of Type 5 installed into the base frame. 


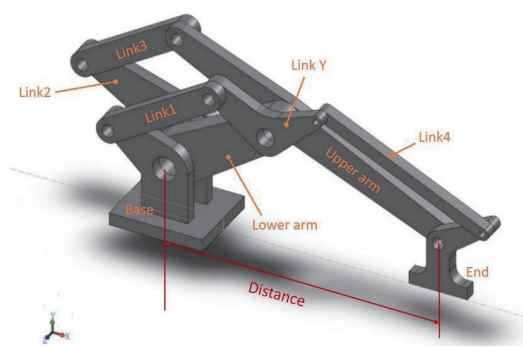

Fig. 3. (Color online) Components of the palletizing robotic arm and their working distances.

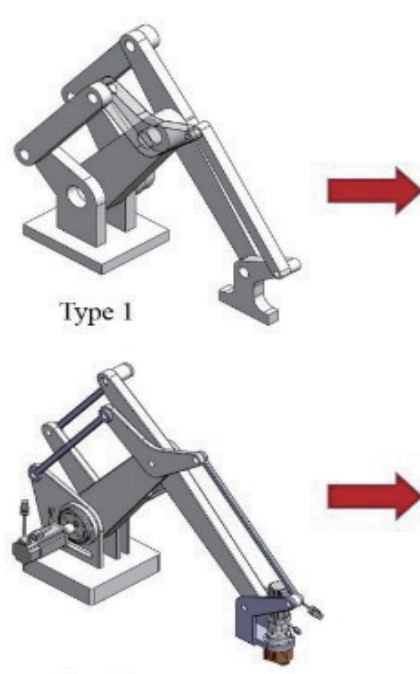

Type 4
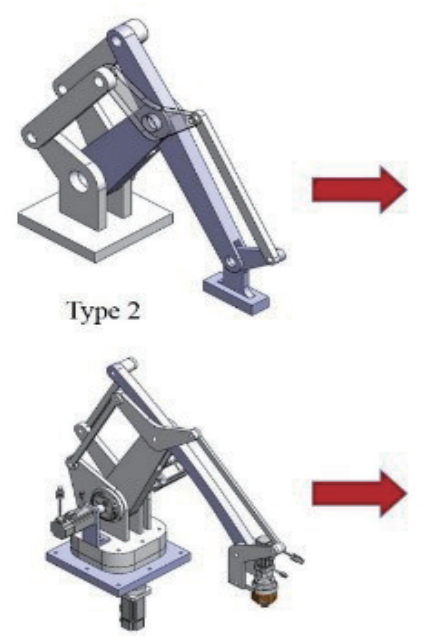

Type 5
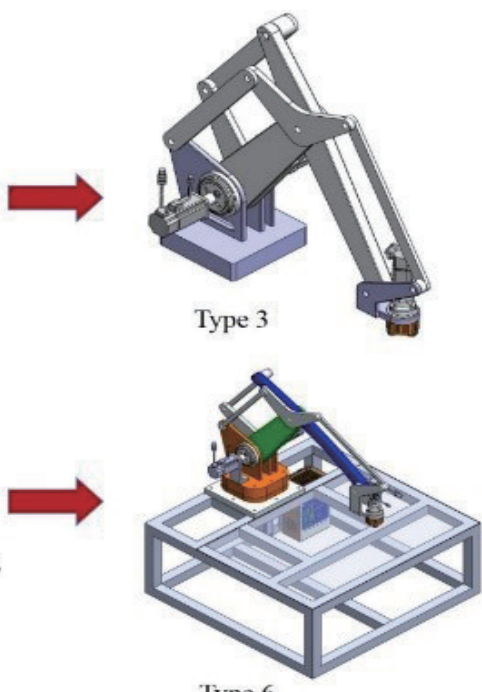

Type 6

Fig. 4. (Color online) Overall robotic arm design at various stages.

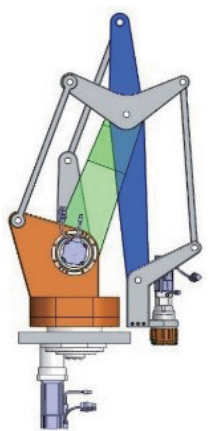

(a)

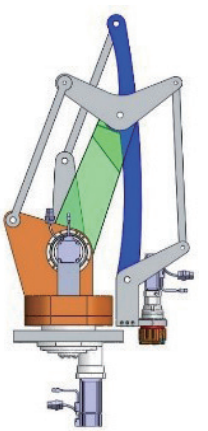

(b)

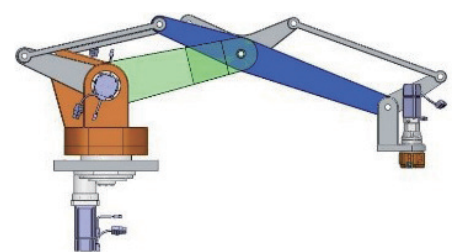

(a)

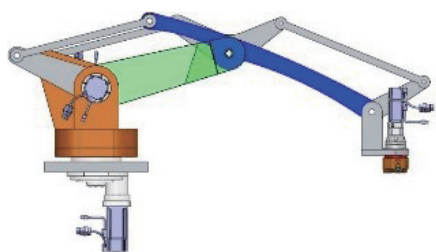

(b)

Fig. 5. (Color online) Changes in shortest distance before and after design change. (a) Shortest distance in Type 4. (b) Shortest distance in Type 5.

Fig. 6. (Color online) Changes in longest distance before and after design change. (a) Longest distance in Type 4. (b) Longest distance in Type 5.

\subsection{Architecture for burr detection}

In this study, an automobile component is used as the research object (Fig. 7). This part is made through stamping, and the part has 40 punching features to be tested. 


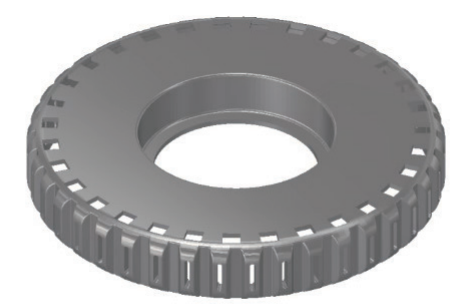

Fig. 7. Inspected object.

An industrial camera with 300000 pixels and backlighting are used as the image-capturing device and light source of the proposed system, respectively, and the images are exported into a computer for preprocessing and to detect burrs after they are captured.

If the value of the grayscale is greater than a threshold value, the grayscale becomes the maximum value of 255 (all white). If the value of the grayscale is less than or equal to the threshold value, the grayscale becomes the minimum value of 0 (all black).

By using an opening function, the image is first eroded and then dilated to remove small and bright details, whereas the remainder of the intensity levels and the high-brightness area are not disturbed. This function is commonly used to remove image noise. A closing function first dilates and then erodes the image, then typically fills small holes or broken lines in the image.

The image coordinates of the first black and white changes within the measurement region are determined, and the relative coordinates of each measurement region are calculated according to their reference number. For example, if the reference number is 1 , the first measurement frame is used as the reference $(0,0)$, and the image coordinates $\left(x_{2}, y_{2}\right)$ in the second measurement frame are subtracted from those in the first measurement frame. For example, with image coordinates $\left(x_{1}, y_{1}\right)$, the relative coordinates obtained are $\left(x_{2}-x_{1}, y_{2}-y_{1}\right)$. The image coordinates are in pixels. This method can measure whether inspection objects are at the same height or whether they are the same distance from each other. When the height of an object is different, the height can be used to calculate whether the height is within the allowable error.

Subtracting the image of the measurement object from that of the standard object for each pixel results in a difference image, which can be used to remove image noise and identify burrs. To investigate whether the vibrations are normal during the grinding process, we use an SVI1000 vibration sensor, manufactured by LNC. This sensor possesses a detection range of $\pm 16 \mathrm{G}$ and can simultaneously detect the vibration values along three axes.

\section{Results and Discussion}

\subsection{Selecting main connecting rod and whole architecture}

The results of our study show that when the magnification coefficient is 1.8 and the lengths of the lower arm, upper arm, and Link2 (see Fig. 3) are 400, 700, and $250 \mathrm{~mm}$, the shortest and longest working distances are 233.06 and $747.58 \mathrm{~mm}$, respectively. The main axis can provide 
360 degree rotation. These working distances meet the standard performance requirements. Therefore, the magnification coefficient and lower arm, upper arm, and Link2 distances presented in Fig. 8 are used. The specifications of the proposed palletizing robot are a cyclic cylinder space with inner radius of $235 \mathrm{~mm}$, outer radius of $745 \mathrm{~mm}$, and height of $300 \mathrm{~mm}$. Figure 9 depicts the proposed whole architecture design, which includes a palletizing robot arm, vision sensor and backlighting source, vibration sensor, and deburring tool. Figure 10 depicts a snapshot of the automatic deburring stage.

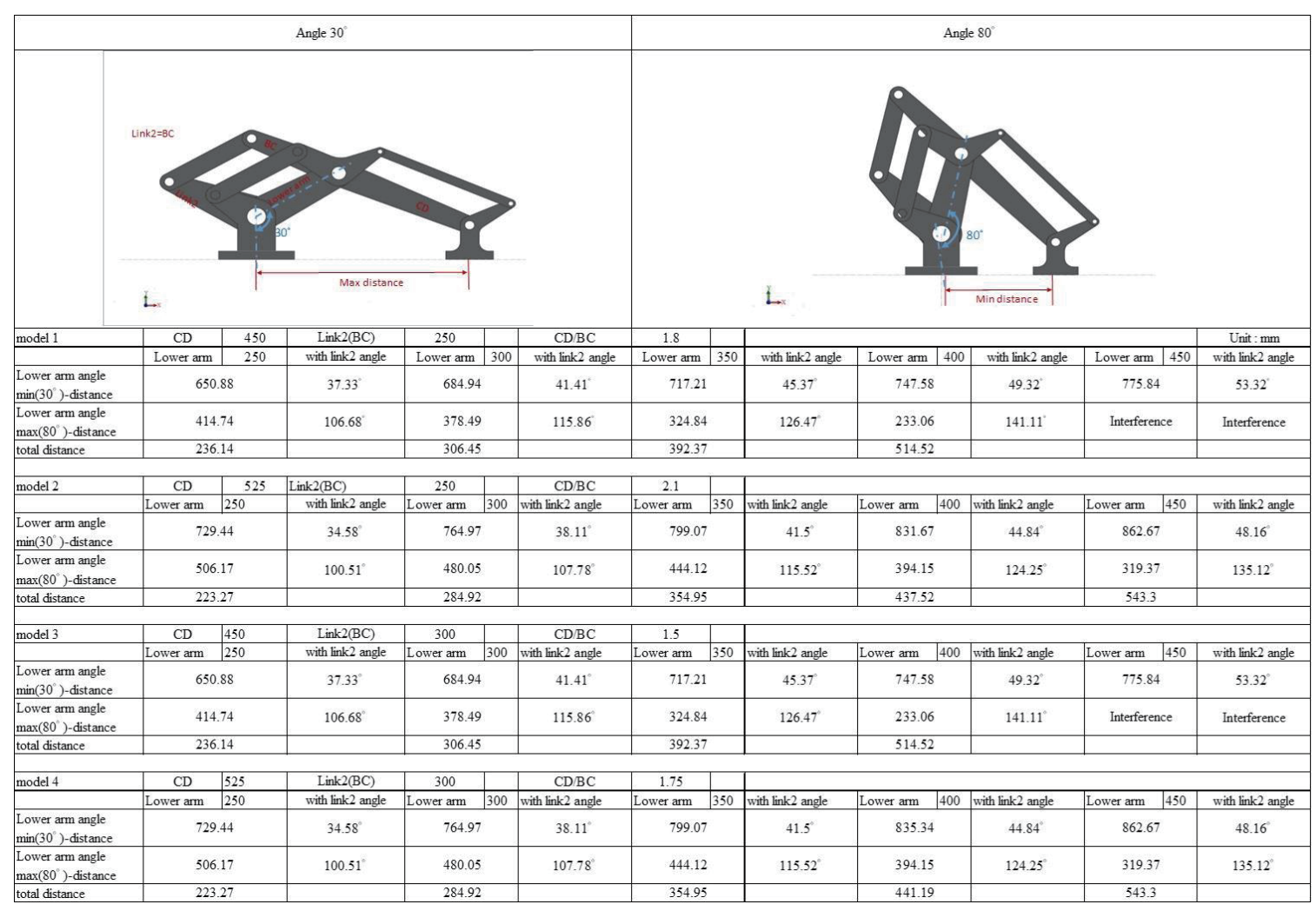

Fig. 8. (Color online) Measurement results for the shortest and longest working distances.

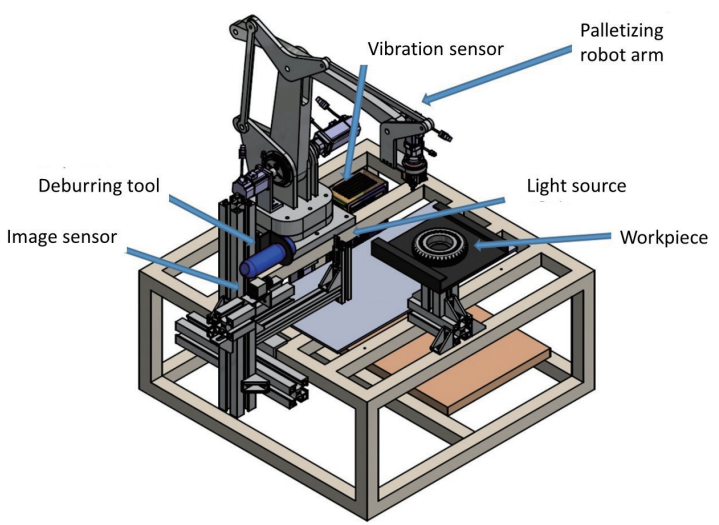

Fig. 9. (Color online) Proposed whole architecture design. 


\subsection{Results of repeatable accuracy test}

For the repeatable accuracy test, the robotic arm moves at a location $20 \mathrm{~mm}$ away from the edge of the table and along the $X$ - and $Y$-axes. The results are measured, and the coordinates are recorded using Delta Robot Automation Studio. The robotic arm is instructed to automatically return to its initial location after performing the programmed actions. The movements are performed 10 times, and the movement data are measured each time. The results are presented in Fig. 11.

\subsection{Automatic rib height measurement}

The image sensor is an IDS 2MP industry camera with image size of $1920 \times 1080$ pixels. Figure 12 depicts a sheet metal forming part mounted on an end bearing for smooth rotation.

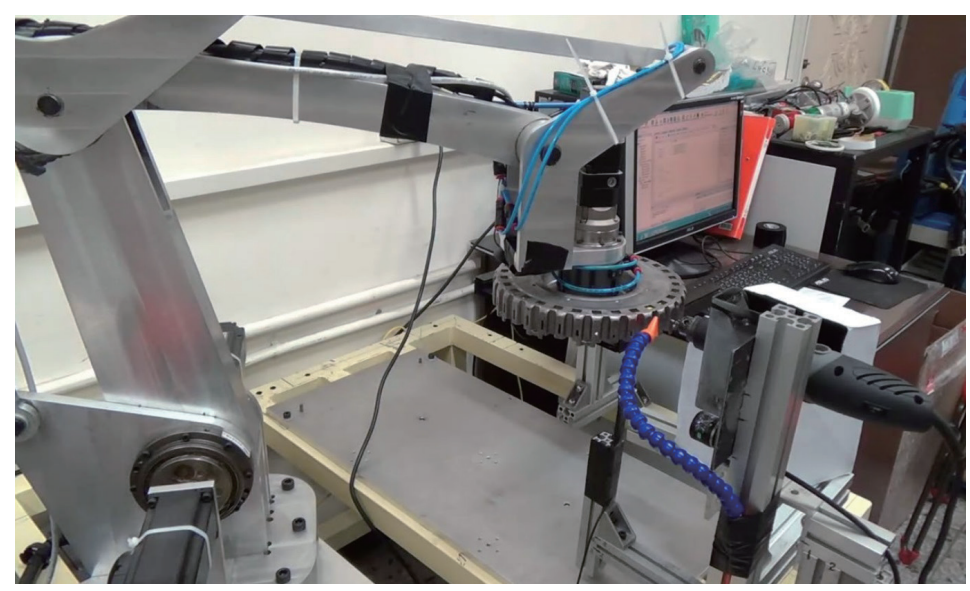

Fig. 10. (Color online) Snapshot of automatic deburring stage.

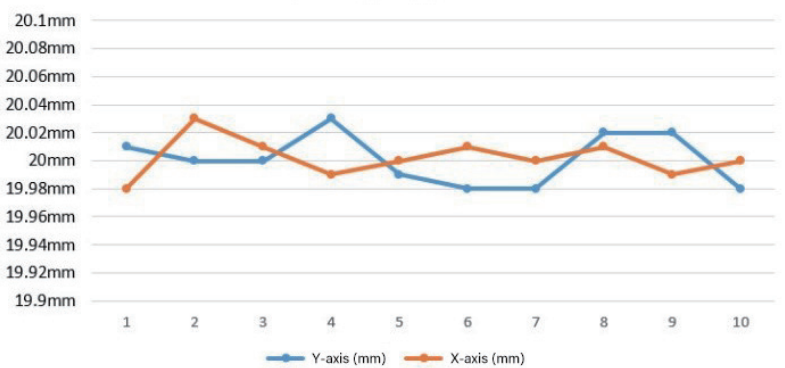

Fig. 11. (Color online) Repeatable accuracy curve for the $X$ - and $Y$-axes.

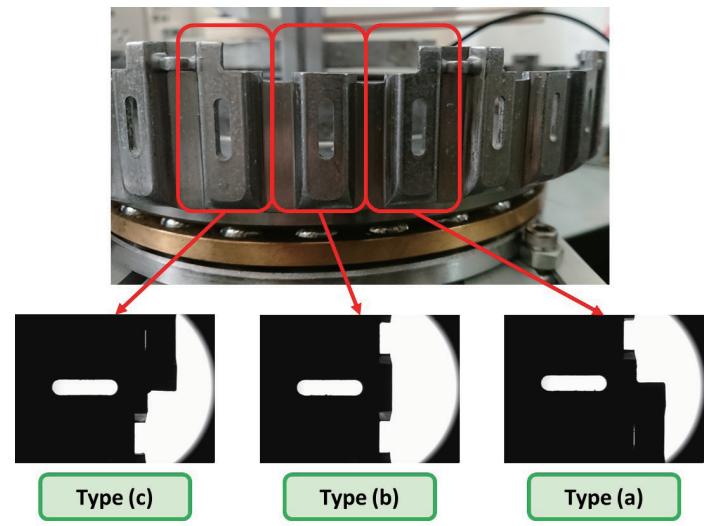

Fig. 12. (Color online) Sheet metal forming parts being measured using three types of rib featureTypes (a), (b), and (c) - captured under backlighting illumination. 
Three types of rib feature-Types (a), (b), and (c) - captured under backlighting illumination are used to obtain a clear image to facilitate measurement. A distinctive hole feature can be detected on each image, which can be treated as a positioning object to improve measurement accuracy.

To obtain accurate height values for comparison, a coordinate measurement machine (CMM) is adopted. Table 1 and Fig. 13 show all 36 portions of the sheet metal forming part measured by the CMM, which can be classified into the three types of rib feature: Types (a), (b), and (c).

To investigate the reliability of the results of the proposed automated optical inspection (AOI) system, we compare the measurement results of the CMM (Table 1) with those of the proposed AOI system (Table 2). Note that the CMM measurement is an absolute measurement method,

Table 1

CMM measurement results for heights of all features in unit of $\mathrm{mm}$.

\begin{tabular}{|c|c|c|c|c|c|c|}
\hline Portion No. & Left & Left high & Middle & Right high & Right & Concave \\
\hline 1 & 28.452 & 30.432 & 30.451 & 35.955 & 36.022 & 32.687 \\
\hline 2 & 28.307 & 30.447 & 30.447 & 30.445 & 28.452 & - \\
\hline 3 & 36.013 & 35.937 & 30.467 & 30.467 & 28.307 & 32.701 \\
\hline 4 & 28.463 & 30.422 & 30.420 & 35.937 & 36.013 & 32.701 \\
\hline 5 & 28.426 & 30.326 & 30.390 & 30.368 & 28.463 & - \\
\hline 6 & 36.014 & 35.928 & 30.417 & 30.326 & 28.426 & 32.576 \\
\hline 7 & 28.456 & 30.356 & 30.443 & 35.927 & 36.014 & 32.576 \\
\hline 8 & 28.469 & 30.369 & 30.399 & 30.366 & 28.456 & - \\
\hline 9 & 35.999 & 35.868 & 30.370 & 30.369 & 28.469 & 35.570 \\
\hline 10 & 28.447 & 30.347 & 30.244 & 35.868 & 35.999 & 32.570 \\
\hline 11 & 28.357 & 30.257 & 30.254 & 30.347 & 28.447 & - \\
\hline 12 & 36.056 & 35.910 & 30.277 & 30.289 & 28.357 & 32.496 \\
\hline 13 & 28.464 & 30.364 & 30.358 & 35.910 & 36.056 & 32.496 \\
\hline 14 & 28.496 & 30.396 & 30.399 & 30.389 & 28.464 & - \\
\hline 15 & 36.117 & 36.016 & 30.515 & 30.396 & 28.496 & 32.628 \\
\hline 16 & 28.591 & 30.491 & 30.448 & 36.016 & 36.117 & 32.628 \\
\hline 17 & 28.528 & 30.528 & 30.563 & 30.491 & 28.591 & - \\
\hline 18 & 36.147 & 36.074 & 30.544 & 30.528 & 28.528 & 32.611 \\
\hline 19 & 28.562 & 30.452 & 30.445 & 36.074 & 36.147 & 32.611 \\
\hline 20 & 28.564 & 30.474 & 30.530 & 30.452 & 28.562 & - \\
\hline 21 & 36.147 & 36.075 & 30.499 & 30.475 & 28.564 & 32.658 \\
\hline 22 & 28.558 & 30.458 & 30.459 & 36.075 & 36.147 & 32.658 \\
\hline 23 & 28.560 & 30.460 & 30.518 & 30.459 & 28.558 & - \\
\hline 24 & 36.119 & 36.046 & 30.506 & 30.460 & 28.560 & 32.656 \\
\hline 25 & 28.556 & 30.456 & 30.432 & 36.039 & 36.118 & 32.656 \\
\hline 26 & 28.520 & 30.420 & 30.480 & 30.431 & 28.555 & - \\
\hline 27 & 36.081 & 36.007 & 30.428 & 30.439 & 28.520 & 32.568 \\
\hline 28 & 28.470 & 30.370 & 30.426 & 30.125 & 36.081 & 32.568 \\
\hline 29 & 28.505 & 30.405 & 30.418 & 30.372 & 28.470 & - \\
\hline 30 & 36.000 & 35.907 & 30.462 & 30.391 & 28.505 & 32.706 \\
\hline 31 & 28.420 & 30.320 & 30.341 & 35.922 & 36.000 & 32.706 \\
\hline 32 & 28.478 & 30.378 & 30.428 & 30.346 & 28.420 & - \\
\hline 33 & 36.006 & 35.899 & 30.426 & 30.359 & 28.478 & 32.716 \\
\hline 34 & 28.505 & 30.405 & 30.424 & 35.937 & 36.006 & 32.716 \\
\hline 35 & 28.511 & 30.411 & 30.486 & 30.418 & 28.505 & - \\
\hline 36 & 36.017 & 35.927 & 30.467 & 30.407 & 28.511 & 32.687 \\
\hline
\end{tabular}




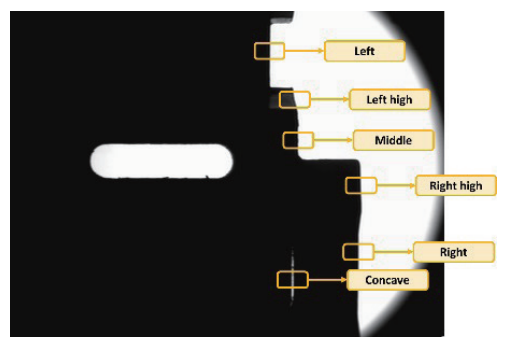

(a)

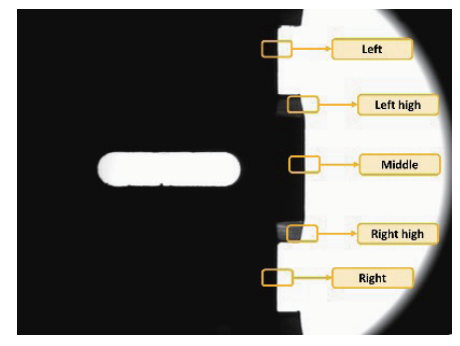

(b)

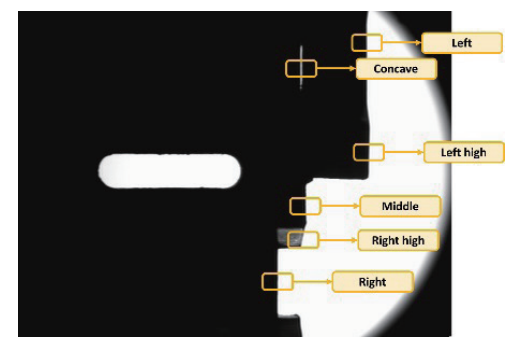

(c)

Fig. 13. (Color online) Three types of rib feature measured by CMM for 36 portions of a whole part. Type (a) Nos. 1, 4, 7, 10, 13, 16, 19, 22, 25, 28, 31, 34. (b) Nos. 2, 5, 8, 11, 14, 17, 20, 23, 26, 29, 32, 35. (c) Nos. 3, 6, 9, 12, 15, 18, 21, 24, $27,30,33,36$.

Table 2

Rib heights measured by the proposed AOI system in unit of pixel.

\begin{tabular}{|c|c|c|c|c|c|c|c|}
\hline Portion No. & $\mathrm{X}_{1}$ & $\mathrm{X}_{2}$ & $\mathrm{X}_{3}$ & $\mathrm{X}_{4}$ & $\mathrm{X}_{5}$ & $\mathrm{X}_{6}$ & $X_{7}$ \\
\hline 1 & 382.729 & 415.500 & 423.734 & 516.016 & 512.637 & 414.426 & - \\
\hline 2 & 383.043 & 417.087 & 422.936 & 417.189 & 382.056 & - & - \\
\hline 3 & 418.985 & 514.040 & 517.492 & 423.938 & 416.987 & 382.690 & 415.846 \\
\hline 4 & 382.887 & 417.500 & 424.216 & 517.784 & 513.633 & 418.512 & - \\
\hline 5 & 381.489 & 416.409 & 422.094 & 417.932 & 381.970 & - & - \\
\hline 6 & 417.598 & 513.694 & 516.667 & 423.764 & 417.500 & 381.035 & 415.227 \\
\hline 7 & 380.890 & 416.500 & 423.029 & 516.627 & 512.636 & 416.765 & - \\
\hline 8 & 380.224 & 415.547 & 421.049 & 416.153 & 380.000 & - & - \\
\hline 9 & 413.500 & 512.737 & 515.653 & 421.169 & 415.038 & 379.187 & 412.211 \\
\hline 10 & 379.846 & 417.500 & 419.120 & 515.238 & 511.583 & 412.122 & - \\
\hline 11 & 379.742 & 417.000 & 417.227 & 413.888 & 378.896 & - & - \\
\hline 12 & 413.500 & 512.277 & 514.681 & 418.927 & 412.564 & 379.012 & 411.992 \\
\hline 13 & 379.768 & 417.500 & 421.048 & 514.932 & 511.687 & 412.500 & - \\
\hline 14 & 379.866 & 414.484 & 419.512 & 414.858 & 378.774 & - & - \\
\hline 15 & 415.500 & 512.048 & 514.983 & 421.996 & 414.139 & 378.869 & 411.847 \\
\hline 16 & 379.945 & 418.500 & 421.519 & 515.112 & 511.350 & 412.520 & - \\
\hline 17 & 379.829 & 414.535 & 420.269 & 415.605 & 378.944 & - & - \\
\hline 18 & 413.500 & 511.973 & 514.918 & 420.799 & 414.098 & 378.353 & 412.628 \\
\hline 19 & 379.090 & 418.500 & 420.286 & 514.405 & 511.275 & 412.406 & - \\
\hline 20 & 379.803 & 414.093 & 419.480 & 414.132 & 378.331 & - & - \\
\hline 21 & 413.841 & 512.602 & 515.692 & 420.982 & 413.565 & 379.180 & 412.715 \\
\hline 22 & 380.090 & 419.500 & 421.076 & 515.972 & 512.556 & 413.393 & - \\
\hline 23 & 380.170 & 414.949 & 420.106 & 415.193 & 379.081 & - & - \\
\hline 24 & 414.500 & 512.541 & 515.913 & 421.628 & 418.500 & 379.889 & 413.941 \\
\hline 25 & 380.188 & 413.500 & 420.910 & 515.604 & 512.001 & 413.012 & - \\
\hline 26 & 380.278 & 415.141 & 420.865 & 414.879 & 379.876 & - & - \\
\hline 27 & 414.500 & 512.647 & 515.592 & 421.105 & 414.126 & 379.783 & 413.080 \\
\hline 28 & 379.899 & 414.068 & 421.000 & 514.688 & 511.154 & 412.690 & - \\
\hline 29 & 379.922 & 414.424 & 419.856 & 414.742 & 378.913 & - & - \\
\hline 30 & 413.500 & 511.589 & 513.996 & 420.352 & 416.500 & 378.982 & 413.234 \\
\hline 31 & 380.073 & 415.500 & 420.680 & 513.805 & 510.578 & 411.986 & - \\
\hline 32 & 380.568 & 414.619 & 419.209 & 413.830 & 378.946 & - & - \\
\hline 33 & 416.820 & 512.176 & 515.544 & 421.567 & 414.671 & 380.784 & 412.909 \\
\hline 34 & 383.201 & 417.500 & 423.069 & 515.975 & 512.021 & 416.406 & - \\
\hline 35 & 383.316 & 418.000 & 422.495 & 417.727 & 382.499 & - & - \\
\hline 36 & 418.967 & 513.993 & 517.649 & 424.149 & 418.500 & 383.147 & 416.049 \\
\hline
\end{tabular}


whereas the AOI system is a relative measurement method. To ensure a fair comparison, we convert each rib feature from an absolute height value to a height value relative to the concave portion. Taking the first image in Type (a) as an example, the relative heights obtained with the CMM and AOI systems based on the concave feature are shown in Tables 3 and 4, respectively.

Figure 14 shows the relative height based on the concave portion obtained from the two systems. It can be observed that the two curves have a difference of about $2 \mathrm{~mm}$ for each feature. The main reason is that the research object is a circular stamping object; because the system only captures images with a single camera and measures them, the measurement values will be distorted because the feature is not in the same image plane. However, the measurement trends of the two systems are similar, indicating that the measurement results of the proposed system are reasonable.

\subsection{Burr detection and deburring}

As mentioned in Sect. 3.3, three types of rib feature-Types (a), (b), and (c)-are captured under backlighting illumination to obtain a clear image to facilitate measurement. Distinctive holes can be detected in each image, which can be treated as objects for positioning to improve measurement accuracy. Burr detection algorithms measure the center of each hole, center the

Table 3

Relative height based on concave portion in unit of $\mathrm{mm}$ (CMM).

\begin{tabular}{lccccc}
\hline Feature & Left & Left high & Middle & Right high & Right \\
\hline Relative value & -4.236 & -2.256 & -2.236 & 3.268 & 3.335 \\
\hline
\end{tabular}

Table 4

Relative height based on concave characteristics in unit of mm (AOI system).

\begin{tabular}{lccccc}
\hline Feature & $\mathrm{X}_{1}-\mathrm{X}_{6}$ & $\mathrm{X}_{2}-\mathrm{X}_{6}$ & $\mathrm{X}_{3}-\mathrm{X}_{6}$ & $\mathrm{X}_{4}-\mathrm{X}_{6}$ & $\mathrm{X}_{5}-\mathrm{X}_{6}$ \\
\hline Relative value & -1.807 & 0.061 & 0.531 & 5.791 & 5.598 \\
\hline
\end{tabular}

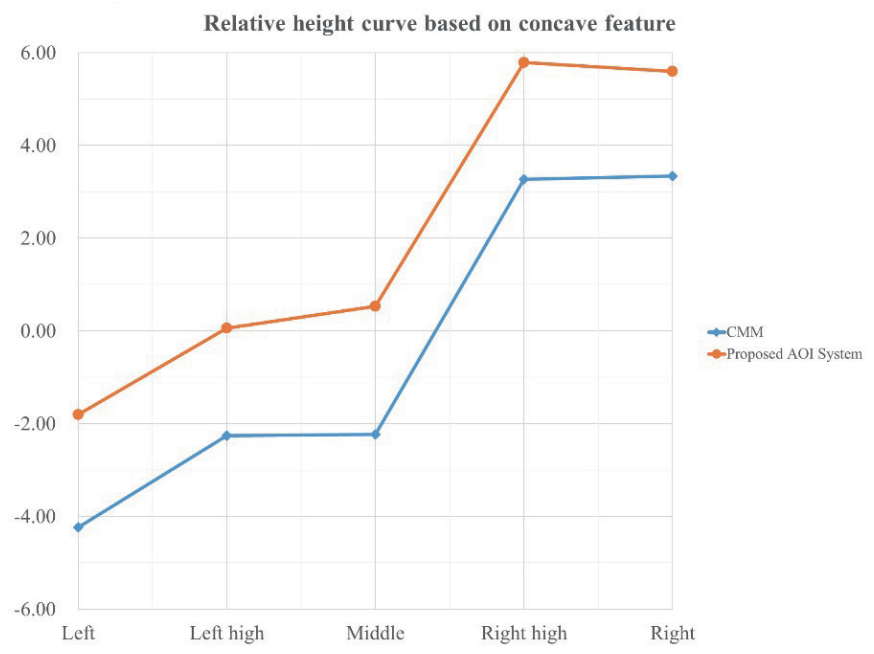

Fig. 14. (Color online) Relative height curves based on concave feature. 
position alignment, subtract images, filter the noise, apply binary thresholding, and conduct blob analysis. Table 5 lists eight detected burrs among 36 holes. Among them, one Type (a) burr, four Type (b) burrs, and three Type (c) burrs are detected. The two largest burrs are both Type (c).

\subsection{Vibration sensor signals}

The LNC SVI-1000 vibration sensor is used in this study, which has 16-bit resolution and a measurement range of $\pm 16 \mathrm{G}$. During the machining process, the vibration sensor control interface detects the vibrations in the $X-, Y$-, and $Z$-directions. When the workpieces are clamped, the highest positive-direction vibration is within $2500 \mathrm{mG}$ (gravitational acceleration of $10^{-3}$ ) and the highest negative-direction vibration is within $-3500 \mathrm{mG}$. In this study, we preset the maximum positive- and negative-direction vibrations as 4000 and $-4000 \mathrm{mG}$, respectively. For vibration signals during the deburring process, the highest positive-direction vibration is within $800 \mathrm{mG}$ and the highest negative-direction vibration is within $-800 \mathrm{mG}$. These vibrations (obtained when the workpieces are clamped) are within the allowable range. For the vibration signals when the palletizing robotic arm drops the workpieces, the highest positive-direction vibration is between 800 and $2400 \mathrm{mG}$, whereas the highest negativedirection vibration is between -800 and $-2400 \mathrm{mG}$, which are within the safety range (as indicated by safety warning signals) and do not affect system operations. Safety warnings are issued when the vibrations exceed the safety range. When this occurs, the system immediately stops operating, which indicates that a malfunction has occurred.

Table 5

(Color online) Burrs among 36 holes detected with the proposed AOI system.

\begin{tabular}{|c|c|c|c|c|c|c|c|}
\hline No. & Portion No. & Type & Capture image & $\rightarrow$ & Standard image & $\rightarrow$ & Detected burr \\
\hline 1 & 4 & (a) & & & & & \\
\hline 2 & 5 & (b) & & & & & \\
\hline 3 & 6 & (c) & & & & & \\
\hline 4 & 8 & (b) & & & & & \\
\hline 5 & 9 & (c) & & & & & \\
\hline 6 & 15 & (c) & & & & & \\
\hline 7 & 20 & (b) & & & & & \\
\hline 8 & 35 & (b) & & & & & \\
\hline
\end{tabular}




\section{Conclusions}

We have developed a palletizing robotic arm that combines an image acquisition device and backlighting to detect and deburr stamped products. The study results are as follows.

1. The palletizing robotic arm features a repeatable accuracy of approximately $0.05 \mathrm{~mm}$.

2. The image processing program developed in this study identifies burrs in workpieces and measures the control sizes of relevant measurement locations.

3. Image subtraction and blob analysis algorithms are used to detect burrs in punching holes. Once the image of the standard component is created, quality assurance for each testing component can be conducted. For burr detection, the results are accurate and fast.

4. In rib height measurement, the AOI results are offset from those of a CMM by approximately $2 \mathrm{~mm}$ but the trend of the results is similar. Therefore, AOI is more suitable for relative measurement.

5. The vibration sensors demonstrate that all the vibrations are within the $4000 \mathrm{mG}$ requirement during workpiece clamping, grinding, and placing. Accordingly, $\pm 4000 \mathrm{mG}$ is set as the value beyond which malfunctioning signals are issued.

\section{References}

1 J. Rejc, J. Cinkelj, and M. Munih: Rob. Comput. Integr. Manuf. 25 (2009) 155. https://doi.org/10.1016/ j.rcim.2007.11.001

2 F. L. Princely and T. Selvaraj: Procedia Eng. 97 (2014) 1906. https://doi.org/10.1016/j.proeng.2014.12.344

3 A. Kuss, M. Drust, and A. Verl: Procedia CIRP 57 (2016) 545. https://doi.org/10.1016/j.procir.2016.11.094

4 L. K. Gillespie: Deburring and Edge Finishing Handbook, M. Dearborn, Eds. (Society of Manufacturing Engineers, New York, 1999) 1st ed., Chap. 1.

5 L. Shahid, F. Janabi-Shjarifi, and P. Keenan: Rob. Comput. Integr. Manuf. 57 (2019) 138. https://doi. org/10.1016/j.rcim.2018.11.009

6 J. C. Aurich, D. Dornfeld, P. J. Arrazola, V. Franke, L. Leitz, and S. Min: CIRP Ann. 58 (2009) 519. https://doi. org/10.1016/j.cirp.2009.09.004

7 Z. Lai, R. Xiong, H. Wu, and Y. Guan: Proc. 2018 IEEE 2nd Int. Conf. Robotics and Biomimetics (IEEE, 2018) 1132.

8 L. Cai and A. A. Goldenberg: Proc. IEEE Int. Conf. Systems, Man and Cybernetics (IEEE, 1989) 787.

9 S. C. Chen and P. C. Tung: Fuzzy Sets Syst. 110 (2000) 341. https://doi.org/10.1016/S0165-0114(98)00190-0

10 S. C. Chen and P. C. Tung: Int. J. Mach. Tools Manuf. 40 (2000) 957. https://doi.org/10.1016/S08906955(99)00099-1

11 G. Seliger, L. H. Hsieh, and G. Spur: CIRP Ann. 40 (1991) 487. https://doi.org/10.1016/S0007-8506(07)62036-8

12 ISO International Standard, Manipulating Industrial Robots-Performance Criteria and Related Test Methods, ISO 9283:1998.

13 M. A. Boboulos: CAD-CAM \& Rapid Prototyping Application Evaluation, http://www.bookBoom.com. (accessed March 2012). 


\section{About the Authors}

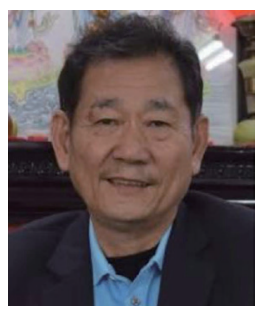

Yung-Fu Hsieh received his EMBA degree from National Sun Yat-sen University, Taiwan. He is a qualified sheet metal technician. His areas of expertise include steel processing, marketing, corporate management, equipment, manufacturing, and installation. Since 2018, he has been a Ph.D. student at National Kaohsiung University of Science and Technology, Taiwan. His research interests are in metal forming, laser cutting, and precision manufacturing. (I108142106@nkust.edu.tw)

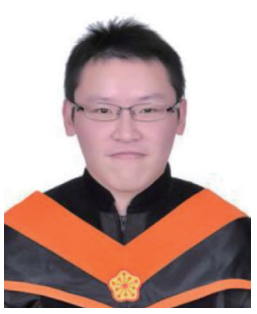

Jhan-Hong Ye received his B.S. and M.S. degrees from National Kaohsiung University of Applied Sciences, Taiwan, in 2014 and 2017, respectively. Since 2017, he has been a Ph.D. student at National Kaohsiung University of Science and Technology, Taiwan. His research interests are in metal forming and computer-aided manufacturing. (jhanhongye@gmail.com)

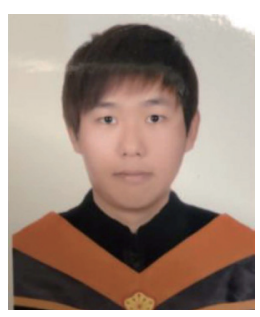

Nan-Jyue Wu received his B.S. degree from I-Shou University and his M.S. degree from National Kaohsiung University of Applied Sciences, Taiwan, in 2011 and 2017, respectively. He has two years of working experience at Kwang Yang Motor Co. Since 2019, he has been working for Taiwan Semiconductor Manufacturing Company. His research interests are in threedimensional measurement, machine vision, and precision manufacturing. (www12458@hotmail.com)

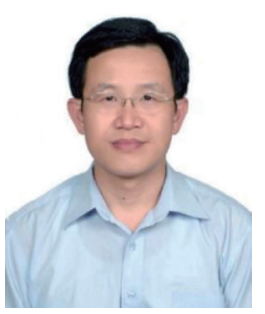

Quang-Cherng Hsu received his B.S., M.S., and Ph.D. degrees from National Cheng Kung University, Taiwan, in 1985, 1987, and 1991, respectively. From 1993 to 1995, he was an engineer at Metal Industries Research \& Development Centre, Taiwan. From 1995 to 2005, he was an associate professor at National Kaohsiung University of Applied Sciences, Taiwan. Since 2005, he has been a professor at National Kaohsiung University of Science and Technology, Taiwan. His research interests are in metal forming, machine vision, and nano-engineering. (hsuqc@nkust.edu.tw) 